Review began 11/19/2021 Review ended 11/25/2021 Published 11/27/202

(c) Copyright 2021

Tasa et al. This is an open access article distributed under the terms of the Creative Commons Attribution License CC-BY 4.0., which permits unrestricted use, distribution, and reproduction in any medium, provided the original author and source are credited.

\section{A Rare Case of Partial Volvulus of the Gall Bladder Detected Preoperatively}

\author{
Anadi S. Tasa ${ }^{1}$, Sandeep Dey ${ }^{2}$, Dhrubajit Gogoi ${ }^{1}$, Vikramjit Baruah ${ }^{2}$ \\ 1. Surgery, Jorhat Medical College and Hospital, Jorhat, IND 2. Anaesthesiology, Jorhat Medical College and Hospital, \\ Jorhat, IND \\ Corresponding author: Sandeep Dey, sandeep.dey09@gmail.com
}

\begin{abstract}
A middle-aged woman with uterine fibroids presented with menorrhagia and diffuse abdominal pain. The patient was anemic, afebrile, anicteric, and had a palpable tender gall bladder. Initial whole abdomen ultrasonography was inconclusive, and computed tomography of her abdomen revealed partial volvulus of the gall bladder. Following optimization, the patient had undergone open cholecystectomy under general anesthesia. Intraoperatively, the gall bladder was distended, with edematous walls and rotated clockwise $\left(270^{\circ}\right)$ along the long axis to the cystic duct. We noted no gangrenous changes, and we performed detorsion of the gall bladder and cholecystectomy. Volvulus of the gall bladder can be associated with high mortality and morbidity. Good clinical examination, a high degree of suspicion given the presentation of the case, proper investigation, and timely management prevented poor outcomes in our case.
\end{abstract}

Categories: Anesthesiology, General Surgery

Keywords: open cholecystectomy, volvulus reduction, general anesthesia, mitral regurgitation, uterine fibroid, gall bladder volvulus

\section{Introduction}

Worldwide, 400 cases of volvulus of gall bladder (GB) have been reported as of 2015 [1], with an estimated incidence of one of 365,000 cases of cholelithiasis [2-6]. Volvulus GB is mainly seen among older women with a median age of 77 years [7]. Most cases are diagnosed intraoperatively [8] and have an associated mortality rate of 6\% [9]. Preoperative diagnosis is complex, and the patient often presents with an acute abdomen [10]. Owing to the pathophysiology of the disease (long or wide mesentery), the anatomical location of the volvulus GB can vary, and it can mimic as acute appendicitis or ischemic bowel [11,12]. We report the case of a patient with vague complaints of diffuse abdominal pain with a palpable lump in the right hypochondrium, which was volvulus GB, on further evaluation.

\section{Case Presentation}

A 50-year-old woman $(47 \mathrm{~kg}$ ) presented with weakness and abdominal pain lasting around two weeks. The pain was gradual in onset, diffuse in nature, was associated with occasional nausea and vomiting. It was not relieved with sleep or food, but only with medication. There was no history of fever, chill, and rigor, burning micturition, yellowish discolouration of the eyes, or urine. The pain abdomen was associated with menorrhagia, lasting for the same duration. She has a similar history of menorrhagia in the past and was diagnosed with uterine fibroids. She had no history of jaundice, tuberculosis. Bladder and bowel habits were regular. There were no significant symptoms suggestive of any systemic disease. On examination, the patient was conscious, cooperative, afebrile, pale, anicteric, and hemodynamically stable. On examination, there was diffuse tenderness on superficial palpation. A lump was palpable in the right hypochondrium, which was tender, intra-abdominal, and moving up and down with respiration. The surface of the lump was smooth, lower margin, medial and lateral margins were palpable, but the upper margin was passing deep to the costal margin. The liver and spleen were not palpable. No other mass was palpable. External genitalia was normal. Per rectal, per vaginal and systemic examination, was normal. On examination, the patient was afebrile, hemodynamically stable, and had a palpable tender GB. Laboratory tests showed severe anemia (hemoglobin, $6.3 \mathrm{~g} / \mathrm{dL}$ ) with unremarkable complete blood count (8200/mm3). Results from liver function test, serum amylase and lipase, random blood glucose, electrocardiogram (ECG), and chest X-ray findings (Figure 1) were within reference ranges. Her rapid antigen test and TrueNat (Molbio Diagnostics Pvt. Ltd., Bangalore, India) chip-based real-time micro-polymerase chain reaction results for beta coronavirus and severe acute respiratory syndrome coronavirus 2 were negative. 


\section{Cureus}

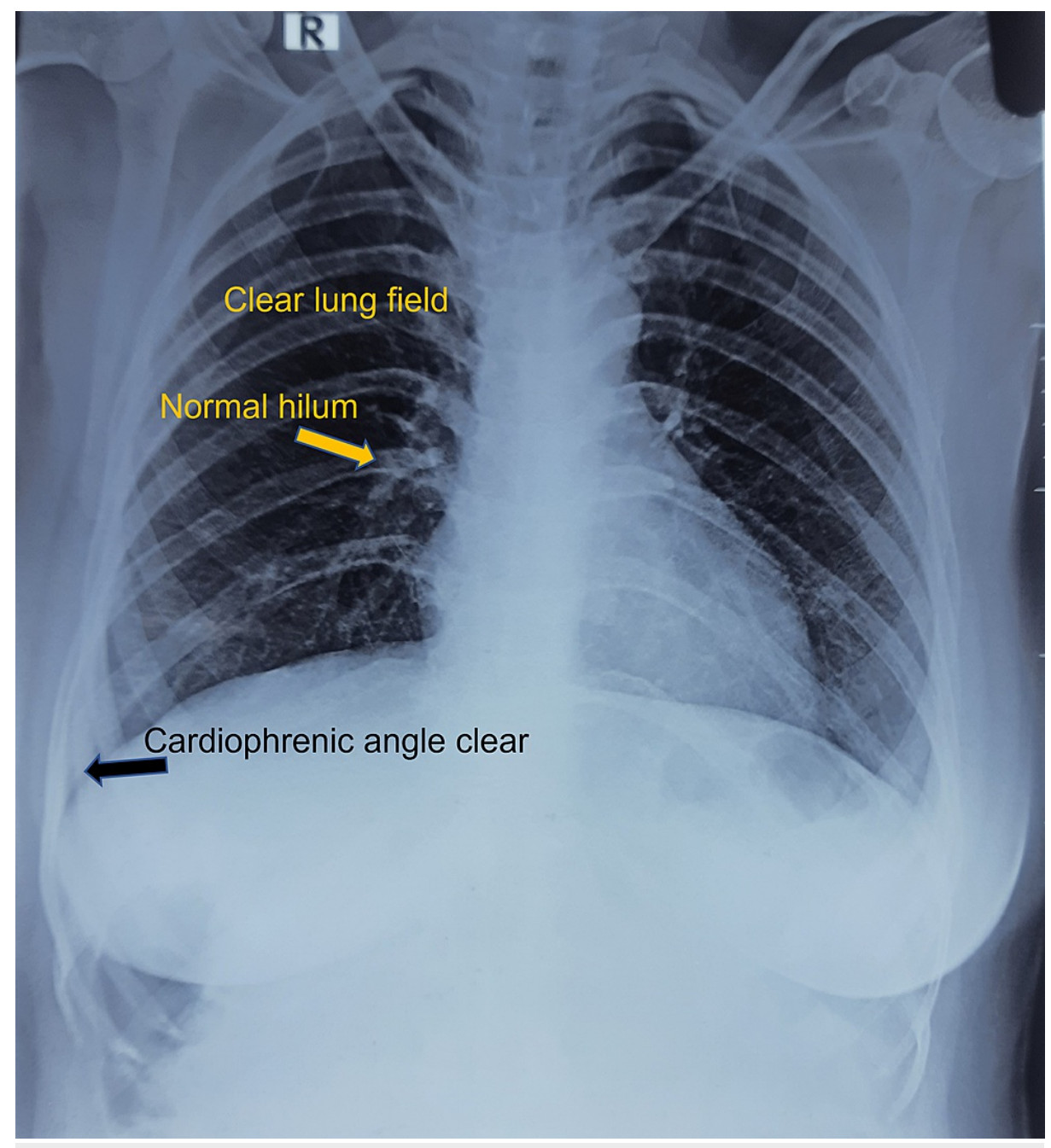

FIGURE 1: Chest X-ray (PA view) showing clear lung fields

PA: posteroanterior.

Abdominal ultrasonography revealed a grossly distended GB with suspicious obstruction at the neck. Common bile ducts and biliary ducts (intrahepatic and extrahepatic) were standard calibers with no evidence of sludge or calculi (Figure 2). 


\section{Cureus}

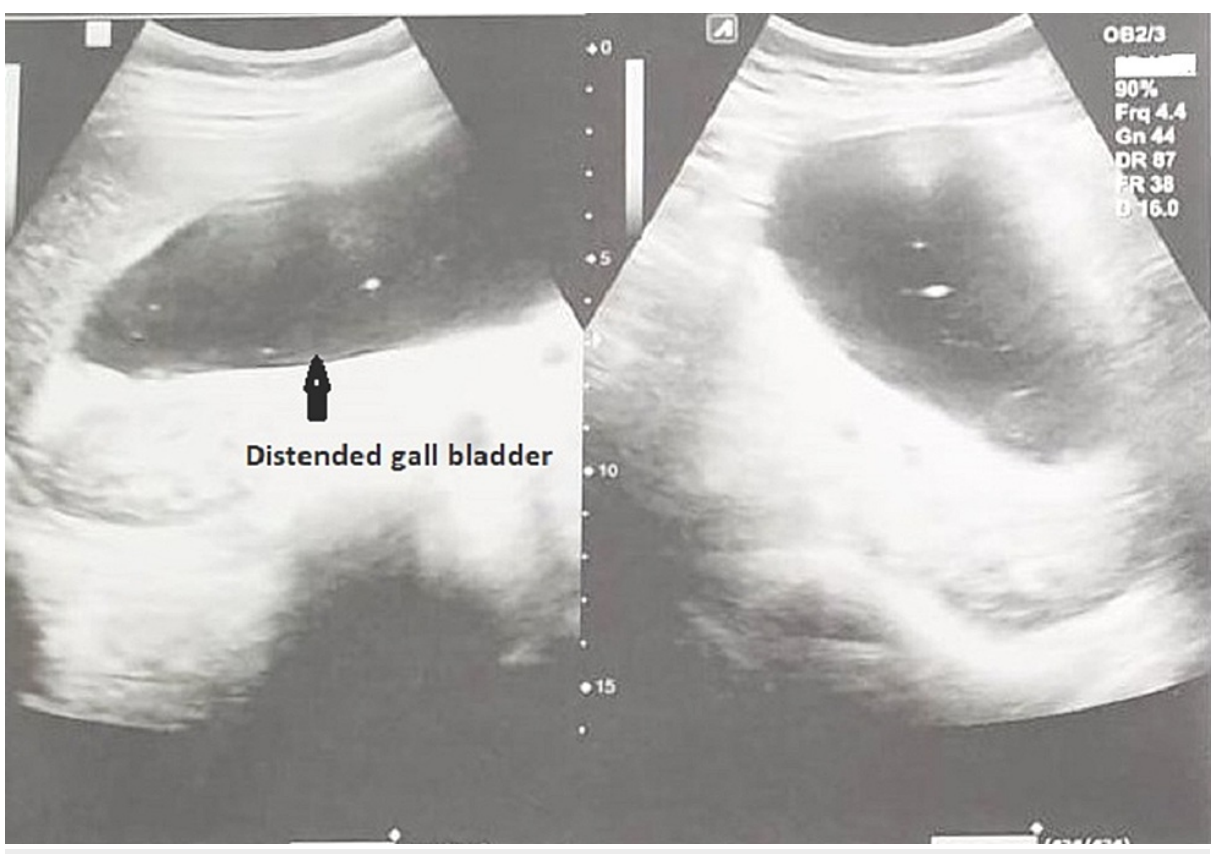

FIGURE 2: Abdominal ultrasonography showing distended gallbladder

Since the patient's abdomen was diffusely tender and not associated with fever or jaundice, a diagnosis other than cholelithiasis/cholecystitis was sought and evaluated. A computed tomography (CT) scan of the abdomen revealed a kink at the GB neck and cystic duct junction, with posterolaterally and inferiorly pulled common hepatic duct. The GB was significantly distended and elongated, suggestive of possible partial volvulus of GB. There was minimal sludge in the GB lumen with no biliary obstruction. There was evidence of intestinal ascariasis. We noted a bulky uterus with multiple intramural myomas (Figure 3).

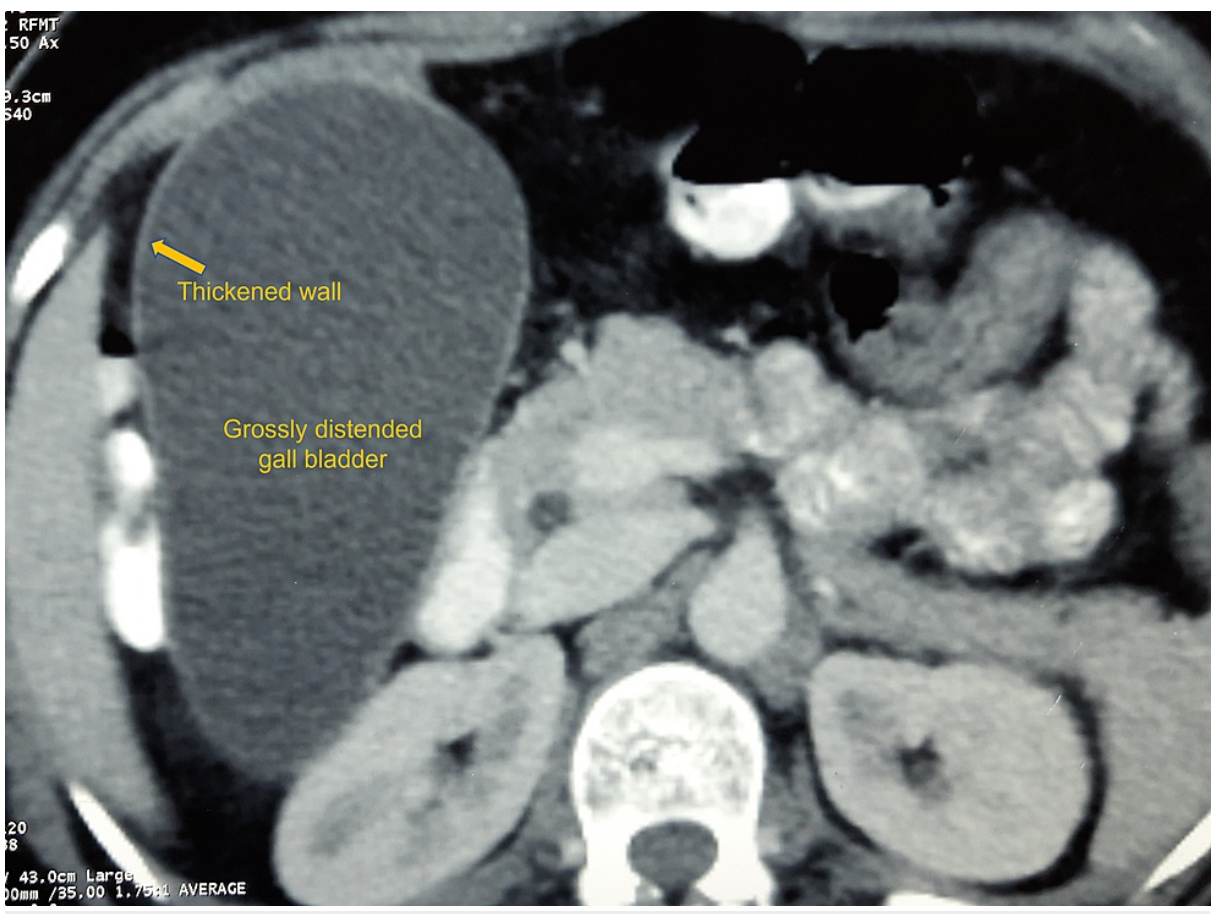

FIGURE 3: Abdominal computed tomography scan

We diagnosed her with uterine fibroid with partial volvulus of the GB and planned an open cholecystectomy under general anesthesia. During her pre-anesthetic check-up, we noted the patient looked ill, pale, anicteric, and she was afebrile, tachycardic (pulse rate: 100 to 110/beats per minute) with blood pressure in the reference range. Her physical status was unremarkable before this episode of abdominal pain, with 
standard exercise tolerance and no history of shortness of breath, orthopnea, paroxysmal nocturnal dyspnea, chest pain (present or past), and palpitation. She had no significant history of tuberculosis, jaundice, or sore throat in the past. Breathing sounds on auscultation were healthy. There was no evidence of crepitation (fine or coarse). The first heart sound was soft, the second heart sound was typical, and we noted no murmur or additional sounds. Examination of the airway revealed Mallampati Grade I and Cormack-Lehane Grade I airway. Other findings were within reference limits.

Two-dimensional echocardiography revealed mild mitral regurgitation (MR) and healthy left ventricular (LV) function. There was no evidence of left atrial or LV hypertrophy with an LV ejection fraction of $73 \%$.

Since the patient's condition did not call for emergency surgery, nor was there any evidence of volume overload or pulmonary congestion secondary to MR, we decided to optimize her before surgery. Three units of packed cell transfusion over three days were given with diuretic coverage, as necessary, to prevent pulmonary congestion. Deworming and mast cell stabilizers were given as there was evidence of intestinal ascariasis on the CT scan abdomen. She received a broad-spectrum intravenous antibiotic. On the night before surgery, she received a mild anxiolytic (alprazolam $0.25 \mathrm{mg}$ ). We planned for elective open cholecystectomy via a right subcostal incision under general anesthesia.

The anesthesia team aimed to maintain preload and avoid bradycardia, hypoxia, hypercarbia, acidosis, and myocardial depression. Standard monitors were attached, including noninvasive blood pressure, pulse rate, oxygen saturation, ECG, and temperature probe. The patient was premedicated with injections of glycopyrrolate $(0.01 \mathrm{mg} / \mathrm{kg})$, palonosetron $(75 \mathrm{mg})$, tramadol $(1.5 \mathrm{mg} / \mathrm{kg})$, and ketorolac $(30 \mathrm{mg}$, intramuscular). We avoided using sedative premedication, which could lead to hypercarbia or increased pulmonary vascular resistance. A nitroglycerine infusion at $5 \mu \mathrm{g} /$ minute was started via pump to improve forward LV stroke volume and decrease the regurgitant fraction. The patient was pre-oxygenated with $100 \%$ oxygen for three minutes and induced with thiopentone ( $4 \mathrm{mg} / \mathrm{kg}$ injection). Atracurium ( $0.5 \mathrm{mg} / \mathrm{kg}$ ) bolus injection was used as a muscle relaxant, and then the patient was intubated. We attached a capnography probe and set mechanical ventilation at 12 breaths/minute to allow sufficient time between breathes for venous return. Anesthesia was maintained using isoflurane inhalation, oxygen, and nitrous oxide, with oxygen maintained at $40 \%$ using an oxygen analyzer and atracurium at the titrated dose. As MR patients are sensitive to small changes in intravascular volume status, we monitored central venous pressure to maintain it between $8 \mathrm{~cm}$ and $12 \mathrm{~cm}$ of water. Also, regular arterial blood gas monitoring was done to avoid hypoxia, hypercarbia, and acidosis that could jeopardize myocardial function. A transdermal ketorolac patch was attached for postoperative analgesia.

At surgery, we noted her GB was grossly distended, edematous, rotated clockwise $\left(270^{\circ}\right)$ around the cystic artery and cystic duct, and without any gangrenous changes (Figure 4, Figure 5). Fortunately, the GB was not perforated by this time. As it was an acute disease without gross contamination of the abdominal cavity, we performed a cholecystectomy through Kocher's incision. The volvulus was reduced, GB was removed, and drains were placed in the right subhepatic space. 


\section{Cureus}

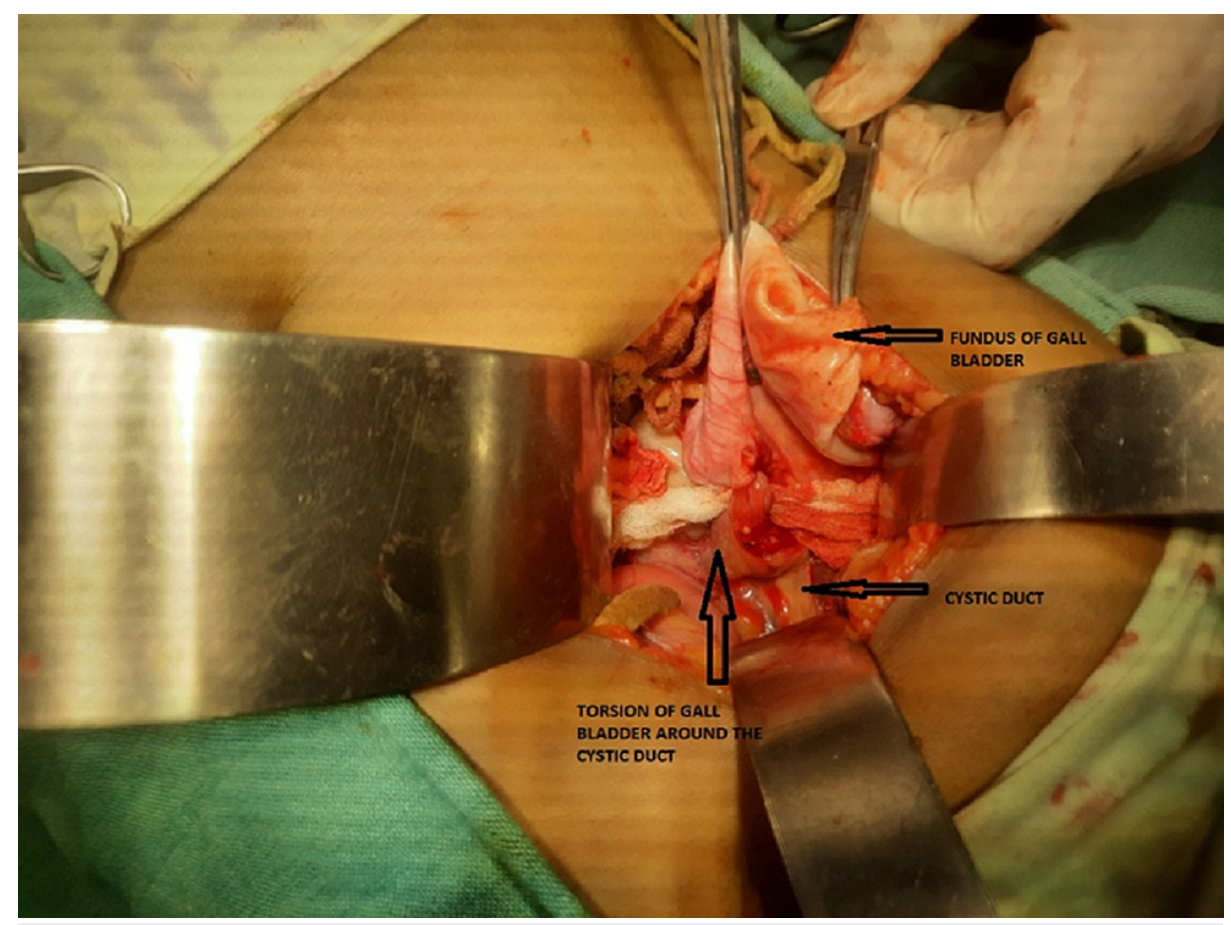

FIGURE 4: Volvulus of gall bladder showing long mesentery with torsion of the gall bladder around the cystic duct and cystic artery

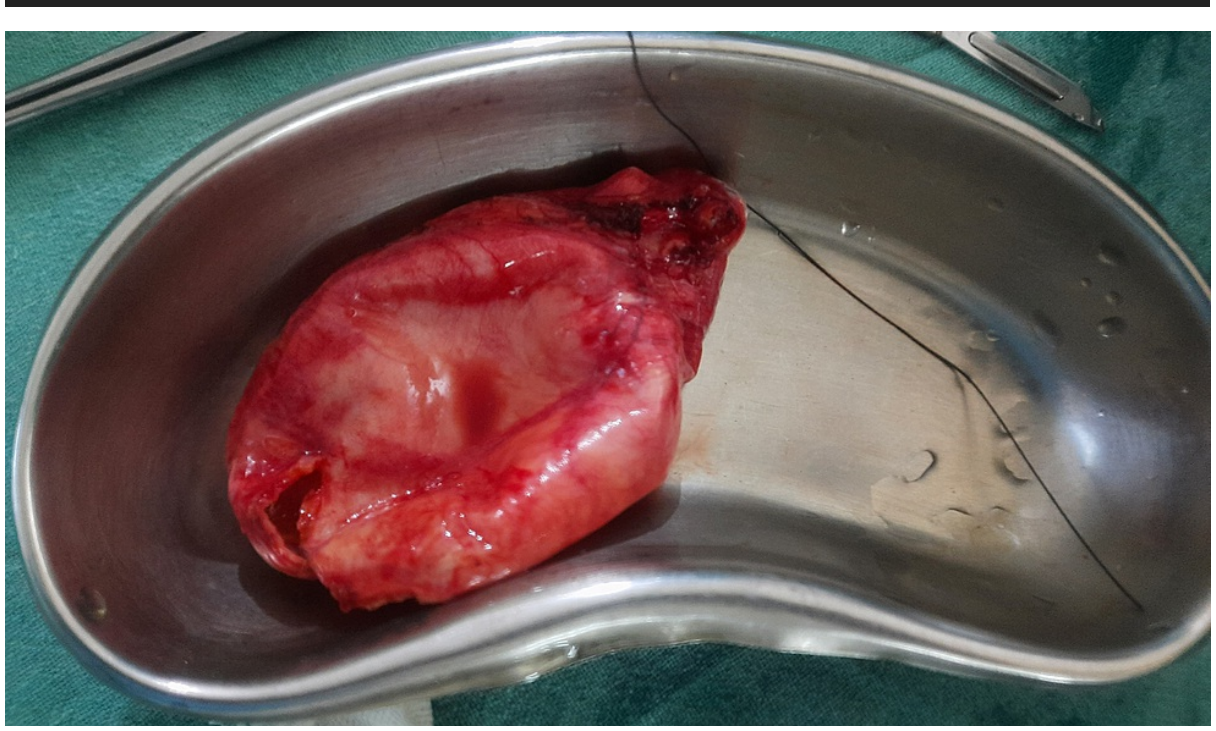

FIGURE 5: Grossly distended, edematous gall bladder with no gangrenous changes

After complete reversal of the neuromuscular blockade, the patient was extubated. Oxygenation was continued in the postoperative care unit, and when the patient had a modified Aldrete score of nine, she was transferred to the postoperative ward. The postoperative period was uneventful. The patient had complete resolution of pain on a postoperative day one, and she was started a regular soft diet. The histopathology reports suggest cholecystitis with areas of erosion, fibrosis, and focal calcification without acute gangrenous changes. The patient was discharged on postoperative day three. At her two-week postoperative follow-up examination, the patient was doing remarkably well. A consulting gynecologist planned to treat the uterine fibroids at a later date surgically.

\section{Discussion}

According to Dayananda et al., Wendel first described the volvulus of the GB in 1898 as a floating GB [13]. 
Predominantly seen in women (1:3 male-to-female ratio), $85 \%$ of the cases occur in patients aged 60 to 80 years [7,14]. Rarely, children as young as two years may be affected [15].

Volvulus of the GB is characterized by clockwise or counter-clockwise torsion of the GB around the longitudinal axis of the cystic duct and cystic artery. The presence of an abnormally long mesentery from the liver bed to the GB, abnormal vascular pedicle to GB, the peristaltic activity of the surrounding bowel, or spinal deformities predisposes a patient to GB twist. Loss of visceral fat with aging contributes to a higher incidence of GB volvulus in older patients $[6,16]$.

Volvulus GB is a diagnostic challenge and needs a high degree of clinical suspicion. Lau et al. in 1982 described the "Triad of Triads" to help identify the features of GB volvulus [17,18]. Even with advances in radiological diagnosis, only five cases of GB volvulus have been diagnosed preoperatively to date [13-15]. Emergency detorsion and cholecystectomy (laparoscopic or open) is the treatment of choice. Delay in treatment can lead to biliary peritonitis from GB rupture and sequelae [19]. Ours is one of the rare cases of GB volvulus diagnosed preoperatively. The patient initially presented with generalized weakness and diffuse, dull, aching abdominal pain with a history of menorrhagia with GB volvulus. Also, there were no signs or symptoms of acute abdomen or cholecystitis (i.e., abdominal colic, fever, jaundice, or vomiting). Our case only fulfilled five of the nine features described by Lau et al. [17,18], with the absence of spinal deformity, right upper quadrant pain, early emesis, and pulse-temperature discrepancy prevented from fulfilling the "Triad of Triads." The diagnosis was guided by clinical examination by a palpable mass in the right hypochondrium on deep abdominal palpation. Timely evaluation and management led to good patient outcomes and home discharge, rather than GB perforation, biliary peritonitis, or a prolonged hospital stay.

\section{Conclusions}

GB volvulus is among the rarer acute abdomen cases attributed to GB pathology, described mainly among older patients. Given the rarity of preoperative diagnosis even with radiological advancement, this case report highlights the importance of clinical examination of all cases of pain abdomen. Even though her symptoms were suggestive of a gynecological cause, abdominal palpation revealed a palpable mass, subsequently diagnosed as volvulus GB. Timely intervention averted the natural course of the disease and patient morbidity.

\section{Additional Information \\ Disclosures}

Human subjects: Consent was obtained or waived by all participants in this study. Conflicts of interest: In compliance with the ICMJE uniform disclosure form, all authors declare the following: Payment/services info: All authors have declared that no financial support was received from any organization for the submitted work. Financial relationships: All authors have declared that they have no financial relationships at present or within the previous three years with any organizations that might have an interest in the submitted work. Other relationships: All authors have declared that there are no other relationships or activities that could appear to have influenced the submitted work.

\section{References}

1. Bhama AR, Ahari A, Chong HS: The diagnostic dilemma of gallbladder volvulus: report of a case . Gen Int Med Clin Innov. 2015, 1:26-9.

2. Escalard C, Calinghen A, Habchi N: Gallbladder volvulus: a rare cause of acute gallbladder distension . J Gastro Hepatol. 2019, 34:1133. 10.1111/jgh.14619

3. Hwang Y, Kulendran K, Ashworth J: Expect the unexpected: torsion of the gallbladder, a rare cause for acute cholecystitis. Cureus. 2018, 10:e3726. 10.7759/cureus.3726

4. Sajid MA, Khan KS, Hanif Z: Diagnostic laparoscopy to investigate unexplained lactic acidosis in critically ill patients - A descriptive single centre cohort study. Ann Med Surg (Lond). 2018, 36:231-4. 10.1016/j.amsu.2018.11.008

5. Aljoe J: Gallbladder volvulus-acute cholecystitis 'with a twist' . J Surg Case Rep. 2018, 2018:rjy303. 10.1093/jscr/rjy303

6. Musthafa S, Aftab Z, Ali SM, Khanna M: Gallbladder volvulus with segmental right liver lobe hypoplasia/atrophy: a preoperative diagnostic dilemma. BMJ Case Rep. 2018, 2018: 10.1136/bcr-2018-224474

7. Tarhan OR, Barut I, Dinelek H: Gallbladder volvulus: review of the literature and report of a case . Turk J Gastro. 2006, 17:209-11.

8. Matsuhashi N, Satake S, Yawata K, et al.: Volvulus of the gall bladder diagnosed by ultrasonography, computed tomography, coronal magnetic resonance imaging and magnetic resonance cholangiopancreatography. World J Gastro. 2006, 12:4599-601. 10.3748/wjg.v12.i28.4599

9. Reilly DJ, Kalogeropoulos G, Thiruchelvam D: Torsion of the gallbladder: a systematic review . HPB (Oxford). 2012, 14:669-72. 10.1111/j.1477-2574.2012.00513.x

10. Janakan G, Ayantunde AA, Hoque H: Acute gallbladder torsion: an unexpected intraoperative finding . World J Emerg Surg. 2008, 3:9. 10.1186/1749-7922-3-9

11. Younan G, Schumm M, Ali F, Christians KK: Gallbladder volvulus in a patient with type I choledochal cyst: a case report and review of the literature. Case Rep Surg. 2016, 2016:1-6. 10.1155/2016/5626531

12. Rueda-Martínez JL, Cascales-Sánchez P, Vázquez-Aragón P, Valero-Liñán AS, Prat-Calero A: Gallbladder 


\section{Cureus}

volvulus: clinical presentation mimicking acute appendicitis of the elderly. Rev Esp Enferm Dig. 2011, 103:656-7. 10.4321/s1130-01082011001200011

13. Dayananda P, Praba RD, Balal MR: Gallbladder volvulus: an uncommon phenomenon: a case report and review of the literature. Clin Med Rev Case Rep. 2018, 5:213. 10.23937/2378-3656/1410213

14. Pottorf BJ, Alfaro L, Hollis HW: A clinician's guide to the diagnosis and management of gallbladder volvulus. Perm J. 2013, 17:80-3. 10.7812/TPP/12-118

15. Mouawad NJ, Crofts B, Streu R, Desrochers R, Kimball BC: Acute gallbladder torsion - a continued preoperative diagnostic dilemma. World J Emerg Surg. 2011, 6:13. 10.1186/1749-7922-6-13

16. Abadía-Barnó P, Coll-Sastre M, Picón-Serrano C, Sanjuanbenito-Dehesa A, Cabañas-Montero J: Gallbladder volvulus: diagnostic and surgical challenges. Cir Cir. 2017, 85:89-92. 10.1016/i.circir.2016.10.013

17. Lau WY, Fan ST, Wong SH: Acute torsion of the gall bladder in the aged: a re-emphasis on clinical diagnosis Aust N Z J Surg. 1982, 52:492-4. 10.1111/j.1445-2197.1982.tb06036.X

18. Ball WR, Dalmia S, Rajamanickam SK, Khan MA: Torted gallbladder causing massive distension and gross necrosis; a rare surgical emergency. BMJ Case Rep. 2013, 2013: 10.1136/bcr-2013-200407

19. Farhat W, Mabrouk MB, Ammar H, et al.: Gallbladder volvulus: a case report and review of the literature . Int J Surg Case Rep. 2019, 60:75-8. 10.1016/j.ijscr.2019.02.025 